\title{
ENT Symptomology in Active COVID-19 Patients in our Tertiary Care Centre
}

\author{
Swapnil Gosavi ${ }^{1}\left(D_{0} \cdot\right.$ Sivasubramaniam Nagarajan $^{1}()^{1} \cdot$ Nirali Jayant Shah $^{1} \cdot$ \\ Neelikattu Aathira Tess Thomas ${ }^{1} \cdot$ Kalpana Rajiv Kumar ${ }^{1}$. Vaishali Sangole ${ }^{1}$
}

Received: 30 April 2021/Accepted: 8 August 2021/Published online: 18 August 2021

(C) Association of Otolaryngologists of India 2021

\begin{abstract}
The aim of this study is to obtain a clear picture of ENT related symptoms in active COVID-19 patients. This study also intends to determine the association of ENT symptoms with Olfactory and Gustatory dysfunction in these patients. Material and Methods: This is a prospective study on 70 active COVID-19 patients who were admitted in the Tertiary Care Hospital. History of all symptoms including Olfactory and Gustatory function were enquired after obtaining Informed Consent. Results: Throat related symptoms was $77.78 \%$, Nasal symptoms accounted for $63.49 \%$ and Ear related symptoms constituted $14.28 \%$. It was seen that Rhinorrhoea and Sneezing was significantly more associated in the presence of Olfactory dysfunction. The most common ENT symptoms were Sore throat (49.21\%), Rhinorrhoea (34.92\%), Sneezing (33.33\%), changes in Smell perception (36.51\%), changes in Taste perception (47.62\%) and Headache (30.16\%). $17.14 \%$ of the patients in this study had both Olfactory and Gustatory dysfunction. Ear related symptoms were also observed where $11.11 \%$ had Aural fullness, $3.17 \%$ with Tinnitus and $6.35 \%$ of the patients complained of Hearing loss which was either noticed for the first time or had worsened on contracting the COVID-19 disease. Discussion: Knowledge of ENT symptomatology in COVID 19 patients will aid in early quarantine and hence limitation of viral transmission. The manifestation of Anosmia or Hyposmia, Ageusia and Aural Fullness in SARS-CoV-2 infected patients can also
\end{abstract}

Sivasubramaniam Nagarajan sivan020393@gmail.com

Swapnil Gosavi gosaviswapnil@gmail.com

1 Department of Otorhinolaryngology, MGM Medical College, Navi Mumbai, Maharashtra, India act as an important tool and help in early isolation and quicker initiation of COVID-19 therapy.

Keywords COVID-19 - Coronavirus - SARS-CoV-2 . ENT symptoms in COVID-19.

Ear problems in COVID-19

\section{Introduction}

Coronavirus disease 2019 (COVID-19) is a contagious respiratory and vascular disease caused by severe acute respiratory syndrome coronavirus 2 (SARS-CoV-2), a specific type of coronavirus. This human RNA coronavirus was isolated in December 2019 in the city of Wuhan, China [1]. In March 2020, World Health Organization (WHO) declared that COVID-19 is a pandemic disease [2]. This infection has high contagious nature of transmission among people and also causes mild or no symptoms in the majority of cases [3].

SARS-CoV-2, the seventh member of the family of Coronaviruses (CoVs) belonging to the family of Coronaviridae, are single-stranded enveloped positive sense RNA viruses having spikes like surface projections and appear like a crown when seen under the electron microscope. Severe Acute Respiratory Syndrome (SARS)-CoV and the Middle East Respiratory Syndrome (MERS)-CoV were other revious outbreaks of coronaviruses (CoVs) [1].

The common general symptoms of the infection are: Fever, dry-cough, sputum production, myalgia, arthralgia, headache, diarrhoea, dyspnoea, fatigue which can lead to Pneumonia, severe Acute Respiratory Distress Syndrome (ARDS) causing death in severe cases. Symptoms regarding Otorhinolaryngology include Pharyngitis/Sore Throat, nasal congestion, rhinorrhoea, earache, aural fullness, 
sensorineural hearing loss, smell and taste disorders in COVID-19 patients [1].

There have been increasing reports that anosmia and dysgeusia are significantly linked to COVID-19 disease. British Rhinology Society literature showed higher viral concentration in the nasal cavity as compared to the throat. Anosmia and dysgeusia have also been reported in paucisymptomatic and asymptomatic COVID-19 positive patient, representing in most cases as the first or the only symptomatology manifestation [4].

The American Academy of Otolaryngology- Head and Neck Surgery stated that anosmia and dysgeusia are reported symptoms of COVID 19 positive patients, hence suggesting that these symptoms must be kept in mind while screening patients with possible COVID-19 infection [5].

Beta-coronaviruses, in which the SARS-CoV-2 belongs, not only involve the respiratory tract but can frequently invade the Central Nervous System (CNS). This has been documented for the SARS-CoV, MERS-CoV, and also in porcine hemagglutinating encephalomyelitis coronavirus $(\mathrm{HEV} 67 \mathrm{~N})$. This suggests that the virus has the property of neuroinvasiveness with many studies highlighting the possible route of spread by olfactory epithelial damage by the virus and its spread to the Central Nervous System [1].

In the ENT region, the cranial nerves which are important for the demonstration of neuroinvasiveness include Olfactory nerve, Trigeminal nerve, Facial nerve and Vestibulo-cochlear nerve for the symptoms of loss of smell, decreased tearing, decreased salivation, loss of taste and reduced hearing/tinnitus. COVID-19 infection could have deleterious effects on cochlear hair cell functions despite being asymptomatic causing typically a virus induced sensorineural hearing loss which occasionally recovers spontaneously [1].

Otolaryngologists, Anaesthetist, and surrounding staff are a high-risk group for COVID-19 infection, as they are exposed to viral transmission directly through mucus and aerosolized particles during clinical examination, surgeries, or other interventions in the head and neck area [6]. Evidence from China, Italy, and Iran and recently from the United Kingdom suggests that otolaryngologists are among the highest risk group of contracting the SARS-CoV-2 virus, especially when their examinations and procedures are performed without using appropriate personal protective equipment (PPE).

ENT clinical examination is considered a high-risk medical procedure for the transmission of the SARS-CoV2 virus. Endoscopic examinations of the nose, sinuses, pharynx, and larynx are considered aerosol-generating procedures. The virus appears to be present in the upper aerodigestive tract, with very high concentrations in the nasal cavity and nasopharynx compared to the rest of the pharynx.
The most effective way in preventing the spread of the disease is early detection and isolation of infected individuals. This study intends to give a clear picture of the ENT symptomatology and clinical signs in ENT related to the symptoms in Active COVID 19 patients. The knowledge of the frequency and severity of symptoms will aid in early quarantine and hence limitation of viral transmission. The knowledge of full range of possible symptoms is important and will aid in preventing delays in diagnosis and delays in isolation of infected patients, hence making it easier to deal with the disease.

\section{Methods}

The study was conducted in a Designated COVID Tertiary Care Hospital with active Covid-19 patients. The selected patients are invited to participate after obtaining their consent in a form designed for this purposes. 70 patients were selected for this study as per the Inclusion and Exclusion criteria.

\section{Inclusion Criteria}

1. Confirmed COVID-19 patients after laboratory performed Reverse Transcription Polymerase Chain Reaction (RT-PCR). The tests are performed as per the methodologies prescribed by Indian Council of Medical Research (ICMR).

2. Patients above 10-70 year of age

3. Patients in mild and moderate category at the time of data collection.

4. Patient should be well oriented.

\section{Exclusion Criteria}

Following patients are excluded from the data collections.

1. Patients with olfactory, gustatory, salivary dysfunctions before the pandemic.

2. Patients with neurodegenerative diseases or with dysfunctions concerning the CNS or the PNS

3. Patients on high flow oxygen therapy with noninvasive ventilation (PEEP, CPAP etc.) as it can alter the physiological hydration of the oral cavity

4. Patients who are in the intensive-care unit at the time of the data collection.

5. Immunocompromised individuals. 


\section{Methodology}

All the tests are carried out during the active phase of the symptomatology from COVID-19 disease. History of all symptoms including Olfactory and Gustatory function was taken from patients admitted in the COVID wards, post RT-PCR positive report from day 0 to day 5 . This will include-

a. General questions (age, sex, religion)

b. COVID 19 status according to the criteria (Mild/ Moderate category)

c. Clinical questions (co-morbidities, general, and ENT symptoms associated with COVID-19 infection)

d. Questions about olfactory function

e. Questions about gustatory function

f. Questions about auditory discomfort with focus on hearing and tinnitus.

\section{Statistical Analysis}

Statistical tools and Statistical tests are used appropriately for proper analysis of the data. The number of patients with and without Olfactory and Gustatory dysfunctions were compared and the $p$-value $\leq 0.05$ (less than 0.05 or $5 \%$ ) was been considered significant.

\section{Results}

A total of 70 patients were included in our study. The mean age of patients was $50.35 \pm 17.41$ years (Range 10-70). There were 37 Males (52.86\%) and 33 Females (47.14\%). 35 patients $(50 \%)$ belonged to both Mild and Moderate category respectively. 37 patients (19 males and 18 females) had comorbidities $(52.86 \%)$ while 33 patients did not have any associated comorbidities (47.14\%). The most prevalent associated comorbidities were Hypertension, Diabetes mellitus type 2 and Hypothyroidism (refer Table 1). All COVID 19 patients, who satisfied the selection criteria, were evaluated for symptoms with respect to the field of Otorhinolaryngology during their course in the COVID Wards.

Out of 70 patients who were included in the study, 63 patients $(90 \%)$ had complained of symptoms pertaining to Otorhinolaryngology while 7 patients (10\%) had no ENT related symptoms.

The ENT symptoms most commonly related to the infection included Sore throat, Rhinorrhoea, Sneezing, changes in Smell perception, changes in Taste perception and Headache. Out of 63 patients who presented with ENT related symptoms, 49 patients had Throat related symptoms including Changes in Taste perception (77.78\%), 40 patients had Nasal symptoms including Changes in Smell perception (63.49\%), 9 patients had Ear related symptoms (14.28\%) like Aural fullness, Hearing loss while 21 patients had symptoms of Headache and Facial heaviness $(33.33 \%)$. Percentage of each Otorhinolaryngology symptom seen in 63 patients having ENT related symptoms has been described in Fig. 1 .

In our study, it was seen that 23 out of 70 patients $(32.86 \%)$ had Olfactory dysfunction related to the infection. A total of 30 patients (42.86\%) reported Gustatory dysfunction where impairment was seen in four taste modalities which included salt, sweet, bitter and sour. 12 patients (17.14\%) had reported both Gustatory and Olfactory dysfunctions. In most of these patients, Gustatory and Olfactory dysfunctions were often associated with Rhinorrhoea, Sneezing, Sore throat, Post nasal discharge or Nasal obstruction episodes. 1 patient had only Olfactory dysfunction and no other ENT related symptoms. Similarly, presence of only Gustatory dysfunction was seen in 3 patients while 2 patients had both Gustatory and Olfactory dysfunction but no other ENT related symptoms. The comparison of Groups with and without Olfactory/Gustatory dysfunction has been done in Tables 2 and 3. It was seen that both Olfactory and Gustatory dysfunctions occurred equally in all age groups and there was no significant difference in the age presentation of these symptoms. All other ENT symptoms were equally associated in patients with and without Olfactory/Gustatory dysfunctions. However, it was seen that Rhinorrhoea and Sneezing was significantly more associated with patients having the presence of Olfactory dysfunction ( $p$ - value of 0.04 and 0.0008 respectively).

\section{Discussion}

Patients infected with SARS-CoV-2 have a wide range of presentation of ENT related symptoms. Studies have also shown that the virus causes disturbance in smell and taste. In this study, we evaluated the frequency of all ENT related symptoms in patients who were admitted in the COVID wards in the active phase of the disease. The frequency of Smell and Taste sensation dysfunctions was also evaluated in these patients. Comparison of patients with and without Olfactory/Gustatory dysfunction was done.

The rapid increase in the number of COVID 19 patients and also the number of deaths due to the infection are a major burden on the healthcare systems. Hence, early detection followed by quick isolation of infected patients can help in breaking the transmission of the disease and decreasing the severity of the disease. Therefore, it is very important to have knowledge of all symptoms caused by the disease [7]. In cases of severe category, patients may 
Table 1 General statistics

\begin{tabular}{ll}
\hline Gender & $37(52.86 \%)$ \\
Males & $33(47.14 \%)$ \\
Females & $50.35 \pm 17.41$ years $($ Range $10-70)$ \\
Age in years & $35(50 \%)$ \\
Mild category & $35(50 \%)$ \\
Moderate category & $37(52.86 \%)$ \\
Comorbidities & $19(27.14 \%)$ \\
Males & $18(25.72 \%)$ \\
Females & 19 out of $70(27.14 \%)$ \\
Hypertension & 24 out of $70(34.28 \%)$ \\
Diabetes mellitus type 2 & 4 out of $70(5.71 \%)$ \\
Hypothyroidism & 2 out of $70(2.85 \%)$ \\
Heart disease & 3 out of $70(4.28 \%)$ \\
Kidney disease & 1 out of $70(1.43 \%)$ \\
Asthma & 1 out of $70(1.43 \%)$ \\
Koch's & 5 out of $70(7.14 \%)$ \\
Others &
\end{tabular}

\section{OTORHINOLARYNGOLOGY SYMPTOMS}

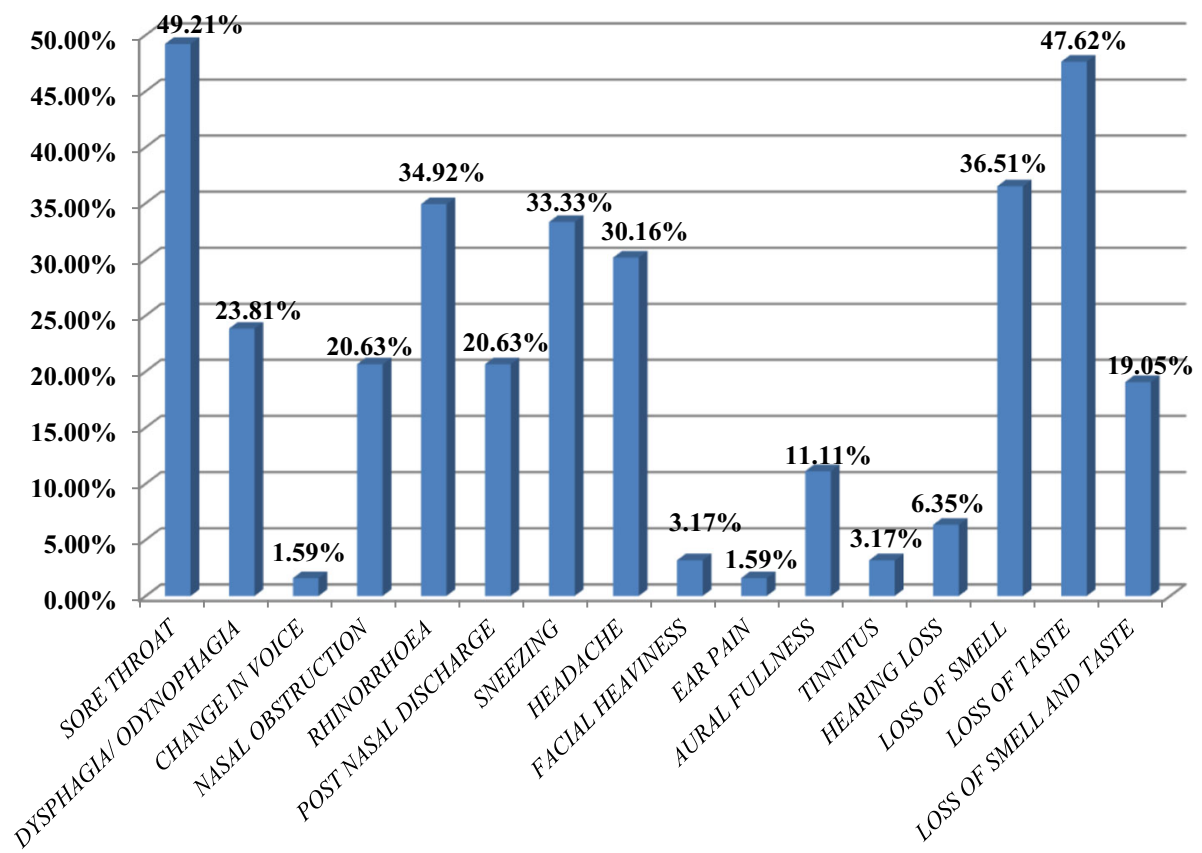

Fig. 1 Otorhinolaryngology symptoms presented by active COVID 19 patients

develop viral pneumonia and acute respiratory distress syndrome (ARDS) and can lead to death despite treatment [8].

In the present study, active COVID 19 patients in mild or moderate category were evaluated for ENT related symptoms. The most common Otorhinolaryngology symptoms were Sore throat $(49.21 \%)$, Rhinorrhoea $(34.92 \%)$, Sneezing (33.33\%), changes in Smell perception
(36.51\%), changes in Taste perception (47.62\%) and Headache $(30.16 \%)$. In the study done by Erdal Sakalli and others, the various frequencies of symptoms concluded were Headache $(56.4 \%)$, Nasal obstruction $(53.5 \%)$, Loss of sense of smell $(51.2 \%)$, Sore throat $(50.2 \%)$, Loss of sense of taste $(47.1 \%)$ and Rhinorrhea (38.5\%) [3].

In a study in Italy conducted on COVID 19 positive patients with mild symptoms, all participants were 
Table 2 Comparison of groups with and without Olfactory dysfunction

\begin{tabular}{llll}
\hline & Presence of olfactory dysfunction & Absence of olfactory dysfunction & $p$ - value \\
\hline Mean age in years & $47.48 \pm 16.85$ years & $51.77 \pm 17.69$ years & 0.33 \\
Sore throat & $34.78 \%$ & $48.94 \%$ & 0.26 \\
Dysphagia/odynophagia & $21.74 \%$ & $21.28 \%$ & 0.96 \\
Nasal obstruction & $21.74 \%$ & $17.02 \%$ & 0.63 \\
Rhinorrhoea & $47.83 \%$ & $23.40 \%$ & $\mathbf{0 . 0 4}$ \\
Post nasal discharge & $21.74 \%$ & $17.02 \%$ & 0.63 \\
Sneezing & $56.52 \%$ & $17.02 \%$ & $\mathbf{0 . 0 0 0 8}$ \\
Headache & $34.78 \%$ & $23.40 \%$ & 0.31 \\
Aural fullness & $17.39 \%$ & $6.38 \%$ & 0.15 \\
\hline
\end{tabular}

The bold values indicate that the particular p-value less than 0.05 or $5 \%$ is significant

Table 3 Comparison of groups with and without Gustatory dysfunction

\begin{tabular}{llll}
\hline & Presence of Gustatory dysfunction & Absence of Gustatory dysfunction & $p$ - value \\
\hline Mean age in years & $51.17 \pm 17.74$ years & $49.75 \pm 17.38$ years & 0.73 \\
Sore throat & $50.00 \%$ & $40.00 \%$ & 0.40 \\
Dysphagia/odynophagia & $26.67 \%$ & $17.50 \%$ & 0.35 \\
Nasal obstruction & $16.67 \%$ & $20.00 \%$ & 0.72 \\
Rhinorrhoea & $40.00 \%$ & $25.00 \%$ & 0.18 \\
Post nasal discharge & $23.33 \%$ & $15.00 \%$ & 0.37 \\
Sneezing & $33.33 \%$ & $27.50 \%$ & 0.60 \\
Headache & $30.00 \%$ & $25.00 \%$ & 0.64 \\
Aural fullness & $13.33 \%$ & $7.50 \%$ & 0.42 \\
\hline
\end{tabular}

questioned about their symptoms over the telephone and the Sino-nasal Outcome Test- 22 (SNOT-22) was done. Here, it was found that the most common symptoms were Fever (55.9\%), Cough (60.4\%) and Fatigue (68.3\%) while the upper respiratory tract symptoms were Nasal Congestion $(41.1 \%)$, Sore throat $(31.2 \%)$ and Smell and Taste sensation Loss $(64.4 \%)$ [9].

Various Physicians and Otorhinolaryngologists in particular have reported Anosmia or Hyposmia as well as Ageusia or Dysgeusia also as symptoms of COVID-19 disease. We also evaluated the frequency of Olfactory and Gustatory dysfunction in this study. In our research, $32.86 \%$ of patients reported Olfactory dysfunction while $42.86 \%$ complained of Gustatory dysfunction. $17.14 \%$ of the patients had both Olfactory and Gustatory dysfunction. In our study, it was also seen that Rhinorrhoea and Sneezing was significantly more associated with patients having the presence of Olfactory dysfunction. A survey done on smell and taste disorders in Milan, Italy found that a $33.9 \%$ of patients had at least one taste or smell dysfunction while $18.6 \%$ of patients had both smell and taste dysfunction [10]. In a study conducted by Yan C. et al., it was noted that $68 \%$ of patients had altered smell and $71 \%$ had altered taste [11]. Jerome R. et al. studied 417 COVID 19 positive patients in mild to moderate category and found that $85.6 \%$ and $88.0 \%$ of patients reported Olfactory and Gustatory dysfunctions respectively [4].

The pathophysiological mechanisms which cause Olfactory and Gustatory dysfunctions in COVID-19 patients are considered to be the tropism of the virus SARS-CoV-2 which enters through the olfactory cells by signal interaction between its spike protein and the ACE 2 protein on nasal epithelium target cells [12]. Two mechanisms of infection considered were infection of neuronal olfactory receptor followed by anterograde transport of virus to the olfactory bulb and diffusion through the channels in olfactory ensheathing cells hence forming an open connection to the Central Nervous System [13].

No reports of blurred vision, subconjunctival hemorrhage, eyelid ecchymoses, conjunctival scarring, keratitis or pseudomembrane formation have been seen in COVID19 patients [14]. SARS-CoV-2 can be detected in RT-PCR swab from the lower eyelid fornices collecting tears and conjunctival secretions [15]. SARS-CoV-2 has also been detected in whole saliva in early stages and also from saliva collected from the duct opening of the salivary glands in 
later stages. It has been shown that ACE-2 positive salivary gland epithelial cells are early targets of SARS-CoV-2 [16].

In this study, $14.28 \%$ had Ear related symptoms which included Aural fullness, Tinnitus, Hearing loss. $11.11 \%$ of the patients had Aural fullness while 3.17\% had Tinnitus. $6.35 \%$ of the patients had Hearing loss which was either noticed for the first time or had worsened on contracting the COVID-19 disease. Chong Cui et al. studied 20 patients and found 3 patients to have Tinnitus which reduced with betahistina [17]. In the study done by Francesco Freni and others showed that COVID-19 infection had deleterious effect on the hair cells in the cochlea and cochlear cell functions, even in patients who presented with mild symptoms [1]. It is still unclear whether COVID-19 disease has effects on the auditory system or not but this research like other studies addresses the possible impact of this novel viral infection on the auditory system.

Acknowledgements We would like to thank the Dean, Hospital Director and all other hospital staff who have helped in the conducting the study smoothly and have also ensured that all precautionary measures are provided. We thank all patients for their cooperation, understanding and willingness towards the objective of the study.

Author Contributions All authors have contributed to the study conception and design. Material preparation, data collection and analysis were performed by SN. The Methodology was discussed and finalized by SG. Supervision and Validation was done by SG. The first draft of the manuscript was written by SN and all authors have put forward their comments on previous versions or prepared drafts of the manuscript. The final manuscript was prepared by all authors and has been read and approved.

Funding No funding or financial support sources were used for this study.

Data Availability Data acquired from patients admitted in COVID Wards. The submitted study and its data are original.

\section{Declarations}

Conflict of interest All authors declare no conflict of interest.

Consent to Participate Informed consent was obtained from all individual participants included in the study.

Consent for Publication Informed Consent was signed by participants regarding the publishing of the data.

Ethical Approval Ethical approval was taken prior to the commencement of the study.

\section{References}

1. Freni F, Meduri A, Gazia F (2020) Symptomatology in head and neck district in coronavirus disease (COVID-19): a possible neuroinvasive action of SARS-CoV-2. Am J Otolaryngol 41(5): 102612

2. WHO Director-General's opening remarks at the media briefing on COVID19 -March 2020

3. Sakalli E, Temirbekov D, Bayri E (2020) Ear nose throat-related symptoms with a focus on loss of smell and/or taste in COVID 19 patients. Am J Otolaryngol 41(6):102622

4. Lechien JR, Chiesa-Estomba CM, Ayad T, Saussez S, Horoi M, Le Bon S D et al (2020) Olfactory and gustatory dysfunctions as a clinical presentation of mild-to-moderate forms of the coronavirus disease (COVID-19): a multicenter European study. European Arch Oto-Rhino-Laryngol 277:2251-2261

5. Vaira LA, Salzano G, Deiana G, De Riu G (2020) Anosmia and ageusia: common findings in COVID-19 patients. Laryngoscope 130(7): 1787

6. Joanna Krajewska (Wojciechowska), Wojciech Krajewski, Krzysztof Zub, Tomasz Zatoński (2020) Review of practical recommendations for otolaryngologists and head and neck surgeons during the COVID-19 pandemic. Auris Nasus Larynx 47(4) $544-558$

7. Guan WJ, Ni ZY, Hu Y et al (2020) Clinical characteristics of coronavirus disease 2019 in China. $N$ Engl J Med 382(18):1708-1720

8. Wu Z, McGoogan JM (2020) Characteristics of and important lessons from the coronavirus disease 2019 (COVID-19) outbreak in China: summary of a report of 72314 cases from the Chinese center for disease control and prevention. JAMA 323:2648

9. Spinato G, Fabbris C, Polesel J et al (2020) Alterations in smell or taste in mildly symptomatic outpatients with SARS-CoV-2 infection. JAMA 323:6771

10. Giacomelli A, Pezzati L, Conti F, Bernacchia D, Siano M, Oreni L et al (2020) Self-reported olfactory and taste disorders in SARS-CoV-2 patients: a cross-sectional study. Clin Infect Dis 26:330

11. Yan CH, Faraji F, Prajapati DP, Boone CE, DeConde AS (2020) Association of chemosensory dysfunction and COVID-19 in patients presenting with influenza-like symptoms. Int Forum Allergy Rhinol 10:1-8

12. Brann DH, Tsukahara T, Weinreb C et al (2020) Non-neuronal expression of SARS-CoV-2 entry genes in the olfactory system suggests mechanisms underlying COVID-19 associated anosmia. Sci Adv 6(31):5801

13. Van Riel D, Verdijk R, Kuiken T (2015) The olfactory nerve: a shortcut for influenza and other viral diseases into the central nervous system. J Pathol 235(2):277-287

14. Wu P, Duan F, Luo C, Liu Q, Qu X, Liang L et al (2020) Characteristics of ocular findings of patients with coronavirus disease 2019 (COVID-19) in Hubei Province. China; JAMA Ophthalmol 138(5):575-578

15. Xia J, Tong J, Liu M, Shen Y, Guo D (2020) Evaluation of coronavirus in tears and conjunctival secretions of patients with SARS-CoV-2 infection. J Med Virol 26:25725

16. To KK, Tsang OT, Chik-Yan Yip C, Chan KH, Wu TC, Chan JMC et al (2020) Consistent detection of 2019 novel coronavirus in saliva. Clin Infect Dis 71(15):841-843

17. Cui C, Yao Q, Zhang D, Zhao Y, Zhang K, Nisenbaum E et al (2020) Approaching otolaryngology patients during the COVID19 pandemic. Otolaryngol Head Neck Surg 163(1):121-131

Publisher's Note Springer Nature remains neutral with regard to jurisdictional claims in published maps and institutional affiliations. 